Moroccan J. of Pure and Appl. Anal. (MJPAA)

Volume 3(2), 2017, Pages 186-198

ISSN: Online 2351-8227 - Print 2605-6364

DOI 10.1515/mjpaa-2017-0015

\title{
A bifurcation result involving Sobolev trace embedding and the duality mapping of $W^{1, p}$
}

\author{
Abdelouahed El KhaliL ${ }^{1, a}$
}

Abstract. We consider the perturbed nonlinear boundary condition problem

$$
\begin{cases}-\Delta_{p} u & =|u|^{p-2} u+f(\lambda, x, u) \text { in } \Omega \\ |\nabla u|^{p-2} \nabla u . \nu & =\lambda \rho(x)|u|^{p-2} u \text { on } \Gamma .\end{cases}
$$

Using the Sobolev trace embedding and the duality mapping defined on $W^{1, p}(\Omega)$, we prove that this problem bifurcates from the principal eigenvalue $\lambda_{1}$ of the eigenvalue problem

$$
\begin{cases}-\Delta_{p} u & =|u|^{p-2} u \text { in } \Omega \\ |\nabla u|^{p-2} \nabla u . \nu & =\lambda \rho(x)|u|^{p-2} u \text { on } \Gamma .\end{cases}
$$

2010 Mathematics Subject Classification. 35J20, 35J40, 35J70, 35J92.

Key words and phrases. $p$-Laplacian operator, nonlinear boundary conditions, Sobolev trace embedding, principal eigenvalue, duality mapping, topological degree, bifurcation phenomenon.

\section{Introduction}

The objective of this article is to study a global bifurcation phenomenon for elliptic problem with nonlinear boundary conditions

$$
\begin{gathered}
-\Delta_{p} u=|u|^{p-2} u+f(\lambda, x, u) \text { in } \Omega \\
|\nabla u|^{p-2} \nabla u . \nu=\lambda \rho(x)|u|^{p-2} u \text { on } \partial \Omega=\Gamma,
\end{gathered}
$$

where $\Omega$ is a bounded domain of $\mathbb{R}^{N}, N \geq 1$, with smooth boundary $\Gamma$. Here $\nabla u . \nu:=\frac{\partial u}{\partial \nu}$ with $\nu$ denotes the unit outward pointing normal vector on $\Gamma$. $\rho$ is a weight function belongs to $L^{r}(\Gamma)$ satisfying some hypotheses to be

Received March 2017 - Accepted June 25, 2018, published online in July 2018.

(C)The Author(s) 2017. This article is published with open access by Sidi Mohamed Ben Abdallah University.

${ }^{1}$ Al-Imam Mohammad Ibn Saud Islamic University (IMSIU),

College of Science,

Department of Mathematics and Statistics

P. O. Box 90950, Riyadh 11623, Saudi Arabia

a e-mail: lkhlil@hotmail.com, alakhalil@imamu.edu.sa. 
specified later. $\sigma$ is the $(N-1)$-dimensional surface measure of $\mathbb{R}^{N-1} \cdot f$ is assumed to be a function satisfying some conditions to be fixed later. $-\Delta_{p} u$ denotes the $p$-Laplacian operator defined by

$$
-\Delta_{p} u=-\operatorname{div}\left(|\nabla u|^{p-2} \nabla u\right), \quad \forall u \in W^{1, p}(\Omega)
$$

which occurs in many models of physical context. Let us mention for example, in: the glaciology, the nonlinear diffusion, the filtration problems, in particular in partial differential equations modeling non-Newtonian fluids, (for more details, see [16] and the references therein). Notice that he condition $(E)$ describes a flux through the boundary $\Gamma$ which depends on the solution itself. In this context and for certain physical background of such conditions, see, e.g., [24]. Note that if $p=2$, the problem $\left(P_{f}\right)-(E)$ becomes linear known as the Steklov problem [5].

Let us mention here that several studies have been devoted to the investigation and extensive attention has been paid to for classical Dirichlet problems involving the $p$-Laplacian operator ( see, e.g., [2, 15, 21, 23]). These results have been extended recently to a nonlinear boundary eigenvalue problem by Bronder and Rossi in [11]. The authors show that the principal eigenvalue $\lambda_{1}$ (i.e., the first positive eigenvalue in the spectrum) of

$$
\begin{aligned}
-\Delta_{p} u & =|u|^{p-2} u \text { in } \Omega \\
|\nabla u|^{p-2} \nabla u . \nu & =\lambda \rho(x)|u|^{p-2} u \text { on } \Gamma,
\end{aligned}
$$

is simple and isolated. Motivated by these results, it is our purpose to consider the perturbed problem $\left(P_{f}-(E)\right.$ related to $\left(P_{0}\right)$ and $(E)$ and to elaborate the bifurcation phenomenon when $\lambda$ is closed to $\lambda_{1}$.

Such kind of problems were studied by several authors, with particular cases of nonlinearity $f$ homogeneous boundary conditions is $u=0$ or $\frac{\partial u}{\partial \nu}$ on $\Gamma$. In fact, e.g., for bifurcation of homogenous Dirichlet problems in regular domains, with other conditions on functions $\rho$ and $f$ were studied, we just remember the works given in [7] and [14]. These results were extended for any bounded domain and $\rho$ is locally bounded in [17] and [18], where the authors considered bifurcation phenomena in the interior domain. However, for bifurcation problem posed on whole space $\mathbb{R}^{N}$, we refer to [19].

Our objective is to improve the conditions on both $\rho$ and $f$, so that we extend the results to our bifurcation phenomenon by using an appropriate Sobolev trace embedding linked to a nonlinear eigenvalue problem where the spectral parameter appears at the boundary condition. Note that the results in this article are original except the situation where where the spectral parameter $\lambda$ occurs in the main equation in $\Omega$ with $f \equiv 0, \rho \equiv 0$ and under the homogenous Neumann or Dirichlet boundary conditions, i.e. $\left.\frac{\partial u}{\partial \nu}\right|_{\Gamma}=0$ or $u_{\left.\right|_{\Gamma}}=0$.

The rest of the paper is organized as follows: We state in Section 2 some assumptions and notations which we use extensively. We discuss in this section the well-posedness of our bifurcation problem will established. In Section 3 , we state and prove some results related to: the duality mapping on the functional framework $W^{1, p}(\Omega)($ Lemma 3.1 and Lemma 3.2) and we prove the compactness of operators involved in the variational setting (Lemma 3.3 and Lemma 3.4), in order to ensure the well definition of the topological degree. Section 4 deals with the proof of our main bifurcation result established by Theorem 4.1 .

\section{Preliminaries}

Let $p>1$ and $\Omega$ be a bounded domain (open and connected) in $\mathbb{R}^{N},(N \geq 1)$ with regular boundary $\Gamma$. Our functional framework space is in the usual Sobolev space $W^{1, p}(\Omega)$ equipped by the norm

$$
\|u\|_{1, p}:=\left[\int_{\Omega}\left(|\nabla u|^{p}+|u|^{p}\right) d x\right]^{\frac{1}{p}}, \quad u \in W^{1, p}(\Omega),
$$

For $p>1,\left(W^{1, p}(\Omega),\|\cdot\|_{1, p}\right)$ is reflexive, separable and uniformly convex Banach space [1].

$X^{*}$ stands the dual of the space $X$ connected by the duality pairing $\langle.,$.$\rangle . We denote by " \rightarrow$ (resp. " $\rightarrow$ ") the convergence in the weak (resp. in the norm) topology.

Let us mention that the norm $L^{p}(\Omega)$ (respectively $\left.L^{p}(\Gamma)\right)$ is denoted $\|u\|_{p, \Omega}\left(\|u\|_{p, \Gamma}\right.$ respectively ). 
2.1. Well-posedness discussion. In order to further explicate our problems, we give in this subsections the definition of the $p$-Laplacian without specific boundary conditions.

If $u \in W^{1, p}(\Omega)$ and $u$ satisfies $P_{0}$ ( i.e., $\Delta_{p} u \in L^{p^{\prime}}(\Omega)$ ), then $|\nabla u|^{p-2} \nabla u \in\left(L^{p^{\prime}}(\Omega)\right)^{N}$. Moreover, we can define the normal derivative $\left.\frac{\partial u}{\partial \nu}\right|_{\Gamma}:=\nabla u . \nu$ on $\Gamma$ in the sense of the trace. Furthermore, we deduce from [25]

$$
|\nabla u|^{p-2} \nabla u . \nu_{\left.\right|_{\Gamma}} \in W^{-\frac{1}{p^{\prime}}, p^{\prime}}(\Gamma)
$$

and for every $v \in W^{1, p}(\Omega)$

$$
\left\langle|\nabla u|^{p-2} \nabla u \nu, v\right\rangle=\int_{\Omega}|\nabla u|^{p-2} \nabla u \nabla v d x+\int_{\Omega} \operatorname{div}\left(|\nabla u|^{p-2} \nabla u\right) v d x
$$

where $p^{\prime}:=\frac{p}{p-1}$ is the Hölder's conjugate exponent and $W^{-\frac{1}{p^{\prime}}, p^{\prime}}(\Gamma)$ is the dual of $W^{\frac{1}{p^{\prime}}, p}(\Gamma)$. Note that (2.1) is equivalent to

$$
\int_{\Gamma}\left(|\nabla u|^{p-2} v \nabla u . \nu\right) d \sigma=\int_{\Omega}|\nabla u|^{p-2} \nabla u \nabla v d x+\int_{\Omega} \operatorname{div}\left(|\nabla u|^{p-2} \nabla u\right) v d x
$$

Now, multiplying both sides of $(E)$ by $v \in W^{1, p}(\Omega)$ and integrating over $\Gamma$, we get

$$
\int_{\Gamma}|\nabla u|^{p-2} v \nabla u \nu d \sigma=\lambda \int_{\Gamma} \rho|u|^{p-2} u v d \sigma .
$$

We conclude from the above that the operator $\Delta_{p}$ acts from the space $\left(W^{1, p}(\Omega),\|\cdot\|_{1, p}\right)$ into its dual $\left(W^{1, p}(\Omega)\right)^{*}$ equipped with the dual norm denoted $\|\cdot\|_{*}$.

Embedding theorems ( see [1], [22]) tell us that the mappings

$$
W^{1, p}(\Omega) \hookrightarrow L^{s}(\Gamma)
$$

are continuous for $1 \leq s \leq p^{*}$ and are compact for $1 \leq s<p^{*}$, where $p^{*}$ is the critical exponent of trace Sobolev embedding

$$
p^{*}=\left\{\begin{array}{lll}
\frac{p(N-1)}{N-p} & \text { if } & 1<p<N \\
\infty & \text { if } \quad N \leq p
\end{array}\right.
$$

and

$$
L^{s}(\Gamma)=\left\{u: \Gamma \rightarrow \mathbb{R}, \quad \int_{\Gamma}|u|^{s} d \sigma<\infty\right\} .
$$

Moreover, the mappings

$$
W^{1, p}(\Omega) \hookrightarrow L^{s}(\Omega)
$$

are continuous for $1 \leq s \leq p^{\circ}$ and are compact for $1 \leq s<p^{\circ}$, where $p^{\circ}$ is the critical exponent of standard Sobolev embedding

$$
p^{\circ}=\left\{\begin{array}{lll}
\frac{p N}{N-p} & \text { if } \quad 1<p<N \\
\infty & \text { if } \quad N \leq p
\end{array}\right.
$$

2.2. Assumptions. Let us formulate the conditions on the perturbation function $f$ and on the weight function $\rho$. $(H C) f: \mathbb{R} \times \Omega \times \mathbb{R} \longrightarrow \mathbb{R}$ is a Carathéodory's function

$$
f(\lambda, x, t)=o\left(|t|^{p-1}\right) \text { when } t \rightarrow 0,
$$

uniformly almost everywhere for $x \in \Omega$ and uniformly with respect to $\lambda$ in bounded interval $\mathbb{R}$.

$(G C) f$ verifies the growth condition.

$$
\exists q \in\left(p, p^{\circ}\right)
$$

for which

$$
\lim _{|t| \rightarrow \infty} \frac{f(\lambda, x, s)}{|t|^{q-1}}=0
$$


uniformly almost everywhere for $x \in \Omega$ and uniformly with respect to $\lambda$ in bounded subset of $\mathbb{R}$.

Throughout the function $\rho: \Gamma \rightarrow \mathbb{R}$ is viewed as an indefinite weight belonging to $L^{r}(\Gamma)$ which may change sign, with $r=r(N, p)$ satisfying the conditions $\rho$ may change sign, with $r=r(N, p)$ verifying the conditions

$$
\begin{cases}r>\frac{N-1}{p-1} & \text { if } \quad 1<p \leq N \\ r \geq 1 & \text { if } \quad N<p\end{cases}
$$

Moreover, we suppose that $\sigma\left(\Gamma_{\rho}^{+}\right) \neq 0$ with $\Gamma_{\rho}^{+}=\{x \in \Gamma ; \rho(x)>0\}$ to ensure the existence of sequence of infinity positive eigenvalues for the problem $\left(P_{0}\right)-(E)$.

2.3. Variational setting. Consider the following operators for $u$ and $v$ in $W^{1, p}(\Omega)$ :

$$
\mathcal{A}, G: W^{1, p}(\Omega) \rightarrow\left(W^{1, p}(\Omega)\right)^{*}
$$

and

defined by

$$
F(\lambda, \cdot): W^{1, p}(\Omega) \rightarrow\left(W^{1, p}(\Omega)\right)^{*}
$$

$$
\begin{gathered}
\langle\mathcal{A} u, v\rangle=\int_{\Omega}|\nabla u|^{p-2} \nabla u \nabla v d x+\int_{\Omega}|u|^{p-2} u v d x \\
\langle G u, v\rangle=\int_{\Gamma} \rho(x)|u|^{p-2} u v d \sigma \\
\langle F(\lambda, u), v\rangle=\int_{\Omega} f(\lambda, x, u) v d x .
\end{gathered}
$$

Here $F(\lambda, \cdot)$ is the Nemytskii operator generated by $f$ through $F(\lambda, u)(x)=f(\lambda, x, u(x))$.

Multiplying both sides of $\left(P_{f}\right)$ by $v \in W^{1, p}$ and integrating over $\Omega$, we get

$$
\int_{\Omega} \operatorname{div}\left(|\nabla u|^{p-2} \nabla u\right) v d x=\int_{\Omega}|u|^{p-2} u v d x+\int_{\Omega} f(\lambda, x, u) v d x .
$$

Inserting (2.5) into (2.2), we obtain

$$
\int_{\Gamma}\left(|\nabla u|^{p-2} v \nabla u . \nu\right) d \sigma=\int_{\Omega}|\nabla u|^{p-2} \nabla u \nabla v d x+\int_{\Omega}|u|^{p-2} u v d x+\int_{\Omega} f(\lambda, x, u) v d x .
$$

Finally, (2.3) and (2.6) state the meaning of a solution of $\left(P_{f}\right)-(E)$. That is,

$$
\int_{\Omega}|\nabla u|^{p-2} \nabla u \nabla v d x+\int_{\Omega}|u|^{p-2} u v d x+\int_{\Omega} f(\lambda, x, u) v d x=\lambda \int_{\Gamma} \rho|u|^{p-2} u v d \sigma .
$$

It is worth that the operator $\mathcal{A}$ is not equal to $\left(-\Delta_{p}+|\cdot|^{p-2}\right.$.) except if boundary conditions (Neumann or Dirichlet) are homogeneous.

Definition 2.1. A solution of $\left(P_{f}\right)-(E)$ is a pair $(\lambda, u)$ in $\mathbb{R} \times W^{1, p}(\Omega)$ such that

$$
\int_{\Omega}|\nabla u|^{p-2} \nabla u \nabla v d x+\int_{\Omega}|u|^{p-2} u v d x=\lambda \int_{\Gamma} \rho|u|^{p-2} u v d \sigma+\int_{\Omega} f(\lambda, x, u) v d x,
$$

holds for every $v \in W^{1, p}(\Omega)$.

Observe that (2.8) turns to say that $u$ is a critical point of the functional

$$
\frac{1}{p}\|u\|_{1, p}^{p}-\frac{\lambda}{p} \int_{\Gamma} \rho|u|^{p} d \sigma-\int_{\Omega} f(\lambda, x, u) d x .
$$

Remark 2.1. (i) In view of $(2.8) u$ is a solution of $\left(P_{f}\right)-(E)$, if and only if

$$
\mathcal{A} u-\lambda G u-F(\lambda, u)=0 \quad \text { in } \quad\left(W^{1, p}(\Omega)\right)^{*}
$$

(ii) $\mathcal{A}$ is odd and $(p-1)-$ homogenuous. 


\section{First results}

now, we regroup some preliminary results related to the duality mappings involving the operator $\mathcal{A}$. We show also the compactness of both other operators $G$ and $F(\lambda, \cdot)$ in order to be able to state the appropriate topological degree for the operator stated in (2.9). These results form the heart of the proof of our aim result formulated by Theorem 4.1.

3.1. On the mapping of duality on $W^{1, p}(\Omega)$. The notion of the duality mapping was introduced by $[6]$ and used by [8]. In order to state the next result, let us recall that the gauge function $\varphi:[0, \infty) \rightarrow[0, \infty)$ with $\varphi(t):=t^{p-1}$, is continuous, strictly increasing with $\varphi(0)=0, \lim _{t \rightarrow \infty} \varphi(t)=\infty$ and normalized because $\varphi(1)=1$.

Lemma 3.1. $\mathcal{A}$ is the duality mapping on $\left(W^{1, p}(\Omega),\|\cdot\|_{1, p}\right)$ associated with $t^{p-1}$.

Proof. See [13].

Corollary 3.1. The operator $\mathcal{A}$ is bijective strongly continuous from $W^{1, p}(\Omega)$ onto $\left(W^{1, p}(\Omega)\right)^{*}$. Furthermore, its inverse is the duality mapping of $\left(W^{1, p}(\Omega)\right)^{*}$ onto $W^{1, p}(\Omega)$ associated to $\varphi^{-1}$ defined by $\varphi^{-1}(t)=t^{p^{\prime}-1}$.

Proof. See [13].

Definition 3.1. We say that $T$ satisfies the condition $\left(S^{+}\right)$, if for any sequence $\left\{u_{n}\right\}$ converging weakly to a certain $u$ in $X$ and $\lim \sup \left\langle T u_{n}, u_{n}-u\right\rangle \leq 0$, then $u_{n} \rightarrow u$ in $X$.

Lemma 3.2. The following assertions

(a) $\mathcal{A}$ is hemicontinuous.

(b)

$$
\langle\mathcal{A} u-\mathcal{A} v, u-v\rangle>0, \quad \forall u \neq v .
$$

(c) $\mathcal{A}$ is bounded and coercive.

(d) $\mathcal{A} \in\left(S^{+}\right)$.

hold.

Proof. See [12] and [13].

\subsection{Compactness result.}

Lemma 3.3. Under the assumptions on the weight $\rho$, the operator $G$ is well defined, compact, odd and $(p-1)$ homogeneous from $W^{1, p}(\Omega)$ to $\left(W^{1, p}(\Omega)\right)^{*}$.

Proof. In order to establish the definition and the compactness of $G$, we split the proof into two steps with three cases for each one with respect to values of exponents $r$ and $p$ linked by (1.1).

Step 1: Definition of G.

Case 1: $1<p<N$ and $r>\frac{N-1}{p-1}$. Let $u, v \in W^{1, p}(\Omega)$. Applying the Hölder's inequality, we obtain

$$
\left.\left|\int_{\Gamma} \rho(x)\right| u(x)\right|^{p-2} u(x) v(x) d \sigma \mid \leq\|\rho\|_{r, \Gamma} \leq\|\rho\|_{r, \Gamma}\|u\|_{s, \Gamma}^{p-1}\|v\|_{p^{*}, \Gamma}
$$

where

$$
\frac{p-1}{s}+\frac{1}{p^{*}}+\frac{1}{r}=1
$$

Thus

Such inequality is true if we take

$$
\frac{p-1}{s}>1-\frac{p-1}{N-1}-\frac{1}{p^{*}}=\frac{p-1}{p^{*}} .
$$

$$
\max (1, p-1)<s<p^{*} .
$$


Then by applying trace embeddings $W^{1, p}(\Omega) \hookrightarrow L^{s}(\Gamma)$ and $W^{1, p}(\Omega) \hookrightarrow L^{p^{*}}(\Gamma)$, there is a constant $C=C(\Omega, p, N)>$ 0 so that (3.1) implies

$$
\left.\left|\int_{\Gamma} \rho(x)\right| u(x)\right|^{p-2} u(x) v(x) d \sigma \mid \leq C\|\rho\|_{r, \Gamma}\|u\|_{1, p}^{p-1}\|v\|_{1, p} .
$$

This ensures the well definition of $G$.

Case 2: $p=N$ and $1<r$. Thus $r^{\prime}$ is finite and $N r^{\prime}<N^{*}=\infty$, and $W^{1, N}(\Omega) \hookrightarrow L^{N r^{\prime}}(\Gamma)$. Moreover we can check that

$$
\frac{1}{N r^{\prime}}+\frac{p-1}{N r^{\prime}}+\frac{1}{r}=1
$$

Then Hölder's inequality gives

$$
\left.\left|\int_{\Gamma} \rho(x)\right| u(x)\right|^{N-2} u(x) v(x) d \sigma \mid \leq\|\rho\|_{r, \Gamma}\|u\|_{N r^{\prime}, \Gamma}^{N-1}\|v\|_{N r^{\prime}, \Gamma},
$$

$\forall u, v \in W^{1, N}(\Omega)$. Thus

$$
\left.\left|\int_{\Gamma} \rho(x)\right| u(x)\right|^{N-2} u(x) v(x) d \sigma \mid \leq C\|\rho\|_{r, \Gamma}\|u\|_{1, N}^{N-1}\|v\|_{1, N} .
$$

Here $C=C(\Omega, N)>0$, where we used the Sobolev trace embedding $W^{1, N}(\Omega) \hookrightarrow L^{N r^{\prime}}(\Gamma)$. Hence we deduce the well definition of $G$ in this case.

Case 3: $p>N$ and $1 \leq r$. Here $p^{*}=\infty$ and then

$$
W^{1, p}(\Omega) \hookrightarrow L^{\infty}(\Gamma) .
$$

Thus $\forall u, v \in W^{1, p}(\Omega)$

$$
\left.\left|\int_{\Gamma} \rho(x)\right| u(x)\right|^{p-2} u(x) v(x) d \sigma \mid<\infty
$$

Therefore. $G$ is well defined.

Step 2: The compactness of G. Consider a sequence $\left\{u_{n}\right\} \subset W^{1, p}(\Omega)$ such that $u_{n} \rightarrow u$ weakly in $W^{1, p}(\Omega)$ for some function $u \in W^{1, p}(\Omega)$. Let us prove that $G u_{n} \rightarrow G u$ in $\left(W^{1, p}(\Omega)\right)^{*}$. That is,

$$
\left\|G u_{n}-G u\right\|_{*} \rightarrow 0, \text { as } n \rightarrow \infty .
$$

In other words, we have to show that

$$
\sup \left|\int_{\Gamma} \rho\left[\left|u_{n}\right|^{p-2} u_{n}-|u|^{p-2} u\right] v d \sigma\right| \rightarrow 0, \text { as } n \rightarrow \infty .
$$

Here the supremum is taken over all $v$ in $W^{1, p}(\Omega)$ with $\left.\|v\|_{1, p} \leq 1\right)$.

Like in step 1, we have to consider three cases.

Case 1: $1<p<N$ and $r>\frac{N-1}{p-1}$. Let $s \in\left(\max (1, p-1), p^{*}\right)$ as in (3.2). Thus from (2.5), by replacing $\left(|u|^{p-2} u\right)$ by the difference $\left(\left|u_{n}\right|^{p-2} u_{n}-|u|^{p-2} u\right)$ in both sides, we obtain

$$
\left\|G u_{n}-G u\right\|_{*} \leq C\|\rho\|_{r, \Gamma}\left\|\left|u_{n}\right|^{p-2} u_{n}-|u|^{p-2} u\right\|_{\frac{s}{p-1}, \Gamma} .
$$

Here $C$ is given by the Sobolev trace injection

$$
W^{1, p}(\Omega) \hookrightarrow L^{p^{*}}(\Gamma) .
$$

Now, the continuity of the Nemytskii's operator $u \mapsto|u|^{p-2} u$ from $L^{s}(\Gamma)$ into $L^{\frac{s}{p-1}}(\Gamma)$, the fact that $u_{n} \hookrightarrow u$ weakly in $W^{1, p}(\Omega)$, and the compactness of the embedding $W^{1, p}(\Omega)$ into $L^{s}(\Gamma)$ (because $s<p^{*}$ ) all combined ensure that $\left|u_{n}\right|^{p-2} u_{n}$ converges strongly to $|u|^{p-2} u$ when $n \rightarrow \infty$. This achieves the proof the first case.

Case 2: $p=N$ and $r>1$. Thus there is $C=C(\Omega, N)$ a positive constant such that (3.5) implies

$$
\left\|G u_{n}-G u\right\|_{*} \leq C\|\rho\|_{r, \Gamma}\left\|\left|u_{n}\right|^{N-2} u_{n}-|u|^{N-2} u\right\|_{N r^{\prime}, \Gamma}^{N-1} .
$$


From the continuity of $u \mapsto|u|^{N-2} u$ from $L^{N r^{\prime}}(\Gamma)$ into $L^{N^{\prime}}(\Gamma)$ and from the compactness of the embedding $W^{1, N}(\Omega)$ into $L^{N r^{\prime}}(\Gamma)$, we deduce the desired result.

Case 3: $p>N$ and $r \geq 1$. In this case $p^{*}=\infty$ and

$$
W^{1, p}(\Omega) \hookrightarrow C(\bar{\Omega}) \cap L^{\infty}(\Omega) .
$$

Because $\Omega$ is regular bounded domain, we deduce from (3.9) that the embedding

$$
W^{1, p}(\Omega) \hookrightarrow L^{\infty}(\Gamma)
$$

is compact.

By a simple calculation we conclude via (3.5) and the embedding (3.10) that

$$
\left\|G u_{n}-G u\right\|_{*} \leq\left. C\|\rho\|_{1, \Gamma} \sup _{x \in \Gamma}|| u_{n}\right|^{p-2} u_{n}-|u|^{p-2} u \mid .
$$

Now since $L^{r}(\Gamma) \hookrightarrow L^{1}(\Gamma)$ and taking into account the compactness of the embedding (3.10), we obtain from (3.11) that

$$
\left.\sup _{x \in \Gamma}|| u_{n}\right|^{p-2} u_{n}-|u|^{p-2} u \mid \rightarrow 0, \quad \text { as } n \rightarrow \infty .
$$

Hence $G$ is compact also in this case.

This achieves the proof of the lemma since $G$ is obviously odd and $(p-1)-$ homogeneous.

Lemma 3.4. $\forall \lambda \in \mathbb{R}, F(\lambda,$.$) is compact, F(\lambda, 0)=0$ and

$$
\lim _{\|u\|_{1, p} \rightarrow 0} \frac{F(\lambda, u)}{\|u\|_{1, p}^{p-1}}=0 \text { in }\left(W^{1, p}(\Omega)\right)^{*} .
$$

Proof. First, it is clear that $F(\lambda, 0)=0, \forall \lambda \in \mathbb{R}$.

Second, note that $p<q<p^{\circ}$ and the injection $W^{1, p}(\Omega) \hookrightarrow L^{q}(\Omega)$ is compact. Thus $L^{q^{\prime}}(\Omega) \hookrightarrow\left(W^{1, p}(\Omega)\right)^{*}$.

We conclude that $F(\lambda,$.$) is compact operator acting from W^{1, p}(\Omega)$ into $\left(W^{1, p}(\Omega)\right)^{*}$ unless to prove that $F(\lambda,$. maps from $W^{1, p}(\Omega)$ into $L^{q^{\prime}}(\Omega)$, is well defined and is continuous. Indeed, both conditions $(H C)$ and $(G C)$ ensure that $\forall \epsilon>0$, there exist two positive reel $\delta=\delta(\epsilon)$ and $M=M(\delta)$ such that for almost everywhere $x \in \Omega$, we get the inequalities:

$$
|f(\lambda, x, t)| \leq \epsilon|t|^{p-1} \text { for }|t| \leq \delta
$$

and

$$
|f(\lambda, x, t)| \leq M|t|^{q-1} \quad \text { for }|t| \geq \delta .
$$

These inequalities are justified by combining both limits in conditions $(H C)$ and $(G C)$ respectively. Let us now prove that $F(\lambda,$.$) is well defined. Indeed, using (3.13) and (3.14) and integrating over \Omega$, we deduce

$$
\int_{\Omega}|f(\lambda, x, u(x))|^{q^{\prime}} d x \leq \int_{\Omega_{\epsilon}^{-}}|f(\lambda, x, u(x))|^{q^{\prime}} d x+\int_{\Omega_{\epsilon}^{+}}|f(\lambda, x, u(x))|^{q^{\prime}} d x,
$$

where $\Omega_{\epsilon}^{-}:=\{x \in \Omega,|u(x)| \leq \delta\}$ and $\Omega_{\epsilon}^{+}:=\{x \in \Omega,|u(x)| \geq \delta\}$. Thus

$$
\int_{\Omega}|f(\lambda, x, u(x))|^{q^{\prime}} d x \leq \epsilon^{q^{\prime}} \delta^{(p-1) q^{\prime}}|\Omega|+M^{q^{\prime}} \int_{\Omega}|u|^{q} d x .
$$

That is,

$$
\int_{\Omega}|F(\lambda, u)|^{q^{\prime}} d x \leq \epsilon^{q^{\prime}} \delta^{(p-1) q^{\prime}}|\Omega|+M^{q^{\prime}} \int_{\Omega}|u|^{q} d x .
$$

From this and the fact that $W^{1, p}(\Omega) \hookrightarrow L^{q}(\Omega)$ we deduce that

$$
\int_{\Omega}|F(\lambda, u)|^{q^{\prime}} d x<\infty .
$$


Then we conclude the well definition of $F(\lambda,$.$) . Consequently it is compact.$

Since $F(\lambda,$.$) acts from W^{1, p}(\Omega)$ into $L^{q^{\prime}}(\Omega)$, we claim now that the limit $(3.12)$ holds in the Lebesque space $L^{q^{\prime}}(\Omega)$. Let us rewrite the right side of (3.12) as

$$
\left|\frac{F(\lambda, u)}{\|u\|_{1, p}^{p-1}}\right|^{q^{\prime}}=\left|\frac{F(\lambda, u)}{|u|^{p-1}}\right|^{q^{\prime}}\left|\frac{|u|^{p-1}}{\|u\|_{1, p}^{p-1}}\right|^{q} .
$$

Let $s>1$ to be fixed later. Applying Hölder's inequality to (3.18), we obtain

$$
\left\|\frac{F(\lambda, u)}{\|u\|_{1, p}^{p-1}}\right\|_{q, \Omega}^{q^{\prime}} \leq\left\|\frac{|F(\lambda, u)|^{q^{\prime}}}{|u|^{(p-1) q^{\prime}}}\right\|_{s, \Omega}\left\|\frac{|u|^{q^{\prime}(p-1)}}{\|u\|_{1, p}^{q^{\prime}(p-1)}}\right\|_{s^{\prime}, \Omega},
$$

where $\frac{1}{s}+\frac{1}{s^{\prime}}=1$. To estimate both factors of the product occurring in the right side of the formula (3.19), we shall use again the expressions (3.13) and (3.14) as in (3.15)

$$
\int_{\Omega}\left|\frac{F(\lambda, u)}{|u|^{p-1}}\right|^{q^{\prime} s} d x \leq \int_{\Omega_{\epsilon^{-}}}\left|\frac{F(\lambda, u)}{|u|^{p-1}}\right|^{q^{\prime} s} d x+\int_{\Omega_{\epsilon}+}\left|\frac{F(\lambda, u)}{|u|^{p-1}}\right|^{q^{\prime} s} d x .
$$

Thus

$$
\int_{\Omega}\left|\frac{F(\lambda, u(x))}{|u|^{p-1}}\right|^{q^{\prime} s} d x \leq \epsilon \int_{\Omega} 1 d x+M^{q^{\prime} s} \int_{\Omega}|u|^{q^{\prime} s((q-p))} d x .
$$

That is,

$$
\int_{\Omega}\left|\frac{F(\lambda, u(x))}{|u|^{p-1}}\right|^{q^{\prime} s} d x \leq \epsilon|\Omega| d x+M^{q^{\prime} s} \int_{\Omega}|u|^{q^{\prime} s((q-p))} d x .
$$

The last estimate is true if the exponent $s$ satisfies

$$
s q^{\prime}(q-p)<p^{\circ}
$$

On the other hand, if we take that the exponent $s$ satisfies also

$$
s^{\prime} q^{\prime}(p-1)<p^{\circ}
$$

then the injection $W^{1, p}(\Omega) \hookrightarrow L^{s^{\prime} q^{\prime}(p-1)}(\Omega)$ is compact and there exists a positive constant $C=$ such that

$$
\left\||u|^{q^{\prime}(p-1)}\right\|_{s^{\prime}, \Omega} \leq C^{q^{\prime}(p-1)}\left\||u|^{q^{\prime}(p-1)}\right\|_{1, p} .
$$

Consequently, (3.21), (3.22), (3.24) and the fact that the embedding $W^{1, p}(\Omega) \hookrightarrow L^{s^{\prime} q^{\prime}(p-1)}(\Omega) \cap L^{s q^{\prime}(q-p)}(\Omega)$ is compact which implies

$$
\frac{F(\lambda, u)}{\|u\|_{1, p}^{p-1}}=0 \text { in } L^{q^{\prime}}(\Omega), \text { as }\|u\|_{1, p} \rightarrow 0 .
$$

Which ensures the required limit (3.12) in $W^{1, p}(\Omega)$, provide that the exponent $s$ verifies the system of the inequations (3.23) and (3.24). For that, a simple calculation yields the following bounds

$$
\frac{p^{\circ}}{p^{\circ}-q^{\prime}(p-1)}<s<\frac{p^{\circ}}{q^{\prime}(q-p)} .
$$

Such $s$ exists because

$$
p^{\circ}-q^{\prime}(p-1)>q^{\prime}(q-p)
$$

which is equivalent to

$$
p^{\circ}>q^{\prime}(q-1)
$$

That is,

$$
p^{\circ}>q
$$

as it is already assumed. This ends the proof. 
3.3. Degree theory. Throughout, let $X$ be a real reflexive Banach space and by $X^{*}$ stands for its dual linked by the duality pairing $\langle.,$.$\rangle .$

Definition 3.2. $T: X \rightarrow X^{*}$ is said demicontinuous, if for all $u \in X$ the functional $v \rightarrow\langle T v, u\rangle$ continuous, that is, $T$ is continuous from $(X$, strong $) \rightarrow\left(X^{*}\right.$, weak).

Let $T \in\left(S_{+}\right)$and demicontinuous. Thus we can define the topological degree $\operatorname{Deg}[T ; D ; 0]$, for $D \subset X$ is a bounded and open set with $T u \neq 0, \forall u \in \partial D$. This degree has the same properties like the Leray-Schauder degree $[9]$.

We define critical points $u_{0} \in X$ are such that $T u_{0}=0$. We say that a critical point $u_{0}$ of $T$ is isolated if there is $\varepsilon>0$ such that for every $u \in B_{\varepsilon}\left(u_{0}\right), \quad T u \neq 0$ if $u \neq u_{0}$. We define the index of $u_{0}$, as the limit

$$
\operatorname{Ind}\left(T, u_{0}\right)=\lim _{\epsilon \rightarrow 0^{+}} \operatorname{Deg}\left[T ; B_{\varepsilon}\left(u_{0}\right) ; 0\right]
$$

Here $B_{\varepsilon}\left(u_{0}\right)$ is the ball of radius $\varepsilon$ and centered at $u_{0}$.

In addition, suppose there is a functional of class $C^{1} \Phi: X \rightarrow \mathbb{R}, \Phi^{\prime}=T$ on $X$. Then we have the following lemmas $[20]$.

Lemma 3.5. if $u_{0}$ is local minimum of $\Phi$ and it is an isolated critical point of $T$, then

$$
\operatorname{Ind}\left(T, u_{0}\right)=1
$$

Lemma 3.6. If $\langle T u, u\rangle>0, \forall u \in X,\|u\|_{X}=R$, then

$$
\operatorname{Deg}\left[T ; B_{R}(0) ; 0\right]=1 \text {. }
$$

Remark 3.1. Observe that every continuous map is also demicontinuous. If $T \in\left(S_{+}\right)$then $(T+K) \in\left(S_{+}\right)$for any compact operator $K: X \longrightarrow X^{*}$.

Remark 3.2. $\lambda$ is an eigenvalue of $\left(P_{0}\right)-(E)$ if and only if, there is $u \in W^{1, p}(\Omega) \backslash\{0\}$ solution of

$$
\mathcal{A} u-\lambda G u=0
$$

Define for each $\lambda$ a family of operators $T_{\lambda}$ acting from $W^{1, p}(\Omega)$ to $\left(W^{1, p}(\Omega)\right)^{*}$ defined by

$$
T_{\lambda} u=\mathcal{A} u-\lambda G u-F(\lambda, u) .
$$

In view of lemmas $3.1,3.2,3.3$, remarks 2.1 and 3.2 , we conclude that for any small $\epsilon>0$, the degree

$$
\operatorname{Deg}\left[T_{\lambda}, B_{\epsilon}(0), 0\right]
$$

is well defined for any $\lambda \in \mathbb{R}$ such that $T_{\lambda} u \neq 0$ for any $\|u\|_{1, p}=\epsilon$.

\section{Bifurcation Result}

First, we note that for every $\lambda \in \mathbb{R}$, the couple $(\lambda, 0)$ is a solution of $\left(P_{f}\right)-(E)$ (called trivial solution).

Definition 4.1. The pair $P=(\bar{\lambda}, 0)$ is called a bifurcation point of $\left(P_{f}\right)-(E)$ if any neighborhood of $P$ in $\mathbb{R} \times W^{1, p}(\Omega)$, contains a nontrivial solution of $\left(P_{f}\right)-(E)$.

The next result states the characterization of bifurcation points.

Proposition 4.1. If $(\bar{\lambda}, 0)$ is a bifurcation point of problem $\left(P_{f}\right)-(E)$, then $\bar{\lambda}$ is an eigenvalue of $\left(P_{0}\right)-(E)$. 
Proof. Fix $\bar{\lambda} \in \mathbb{R}$. Since $(\bar{\lambda}, 0) \in \mathbb{R} \times W^{1, p}(\Omega)$ is a bifurcation of $\left(P_{f}\right)-(E)$ there is $\left\{\left(\lambda_{j}, u_{j}\right)\right\}_{n} \subset \mathbb{R} \times\left(W^{1, p}(\Omega) \backslash\{0\}\right)$ of nontrivial solutions of $\left(P_{f}\right)-(E)$ such that

$$
\lambda_{j} \rightarrow \bar{\lambda} \text { in } \mathbb{R} \text { and } u_{j} \rightarrow 0 \text { in } W^{1, p}(\Omega) .
$$

$\left(\lambda_{j}, u_{j}\right)$ are solutions of $(2.4)$. Thus thanks to $(p-1)$-homogenetity, we deduce

$$
\mathcal{A} v_{j}-\lambda_{j} G v_{j}=\frac{F\left(\lambda_{j}, u_{j}\right)}{\left\|u_{j}\right\|_{1, p}^{p-1}}
$$

where $v_{j}=\frac{u_{j}}{\left\|u_{j}\right\|_{1, p}^{p-1}}$. Then the sequence $\left\{v_{j}\right\}_{j}$ is bounded in $W^{1, p}(\Omega)$. Hence for a subsequence if necessary, $v_{j} \rightarrow v$ in $W^{1, p}(\Omega)$, for certain function $v \in W^{1, p}(\Omega)$. Now, using Remark 2.1, Lemma 3.3 and Lemma 3.4, we conclude that

$$
0=\mathcal{A} v_{j}-\lambda_{j} G v_{j}-\frac{F\left(\lambda_{j}, u_{j}\right)}{\left\|u_{j}\right\|_{1, p}^{p-1}} \rightarrow \mathcal{A} v-\bar{\lambda} G v
$$

Consequently,

$$
\mathcal{A} v=\bar{\lambda} G v \text { in }\left(W^{1, p}(\Omega)\right)^{\prime} .
$$

The fact that $v \in W^{1, p}(\Omega) \backslash\{0\}$, (4.3) establishes that $\bar{\lambda}$ is an eigenvalue of $\left(P_{0}\right)-(E)$ in view of Remark 3.2. This completes the proof.

In order to prove our main bifurcation result, we consider the variational characterization of $\lambda_{1}$ setting by

$$
\begin{aligned}
\lambda_{1} & =\min \left\{\frac{\|u\|_{1, p}^{p}}{\int_{\Gamma} \rho(x)|u|^{p} d \sigma} ; u \in W^{1, p}(\Omega), \int_{\Gamma} \rho(x)|u|^{p} d \sigma>0\right\} \\
& =\min \left\{\|u\|_{1, p}^{p} ; u \in W^{1, p}(\Omega), \int_{\Gamma} \rho(x)|u|^{p} d \sigma=1\right\} .
\end{aligned}
$$

In addition, recall for our problem $\left(P_{0}\right)-(E)$, that $\lambda_{1}$ is simple, isolated in the spectrum and it is the unique positive eigenvalue associated to a nonnegative eigenfunction [11].

Consider the product space $\mathcal{E}:=\mathbb{R} \times W^{1, p}(\Omega)$ endowed with the norm

$$
\|(\lambda, u)\|:=\left(|\lambda|^{2}+\|u\|_{1, p}^{2}\right)^{\frac{1}{2}} .
$$

Definition 4.2. The set

$$
\mathcal{C}=\left\{(\lambda, u) \in \mathcal{E}:(\lambda, u) \text { solves }\left(P_{f}\right)-(E), u \neq 0\right\}
$$

is said a continuum of nontrivial solutions of $\left(P_{f}\right)-(E)$, if it is a connected subset in $\mathcal{E}$.

Theorem 4.1. Under $(H C)$ and $(G C)$, the couple $\left(\lambda_{1}, 0\right)$ is a bifurcation point of $\left(P_{f}\right)-(E)$. In addition, there exists a continuum $\mathcal{E}$ of nontrivial solutions of $\left(P_{f}\right)-(E)$ such that $\left(\lambda_{1}, 0\right) \in \overline{\mathcal{C}}$ and $\mathcal{C}$ is either unbounded or there exists $\bar{\lambda} \neq \lambda_{1}$, an eigenvalue of $\left(P_{o}\right)-(E)$, with $(\bar{\lambda}, 0) \in \overline{\mathcal{C}}$.

Proof. We shall employ the classical global bifurcation stated in Theorem 1.3 of [27] and a variation of the technique of ([17], Th. 3.2). So, we may conclude that

$$
\operatorname{Deg}\left[\mathcal{A}-\lambda G ; B_{\epsilon}(0), 0\right]
$$

leaps from -1 to 1 when $\lambda$ is near to the value of $\lambda_{1}$. This is the key point of the proof. Since $\lambda_{1}$ is isolated in the spectrum, we can find for each $\lambda$ near to $\lambda_{1}, \delta>0$ such that the intersection of the interval $\left(\lambda_{1}-\delta, \lambda_{1}+\delta\right)$ with spectrum of $\left(P_{0}\right)-(E)$ is empty. Thanks to (3.4), Lemma 3.6 implies that for $\lambda$ on the left of $\lambda_{1}$

$$
\operatorname{Deg}\left[\mathcal{A}-\lambda G ; B_{\epsilon}(0), 0\right]=1,
$$

Now, we need to compute the value of (4.5) for $\lambda$ on the right of $\lambda_{1}$. Let us construct a function $h: \mathbb{R} \longrightarrow \mathbb{R}$ by

$$
h(t)= \begin{cases}0 & \text { if } t \leq 1 \\ a(t-2) & \text { if } t \geq 3,\end{cases}
$$


with $\frac{\delta}{\lambda_{1}}<a$ and so that $h>0$ and $h^{\prime \prime}>0$ on the interval $(1,3)$. Consider for each fixed $\lambda$ the functional

$$
\begin{aligned}
\Phi_{\lambda}(u) & =\frac{1}{p}\|u\|_{1, p}^{p}-\frac{\lambda}{p} \int_{\Gamma} \rho(x)|u|^{p} d \sigma+h\left(\frac{1}{p}\|u\|_{1, p}^{p}\right) \\
& =\frac{1}{p}\langle\mathcal{A} u, u\rangle-\frac{\lambda}{p}\langle G u, u\rangle+h\left(\frac{1}{p}\|u\|_{1, p}^{p}\right) .
\end{aligned}
$$

Thus $\Phi_{\lambda}$ is of class $C^{1}$. We can also show that a critical point $u_{0} \in W^{1, p}(\Omega)$ of $\Phi_{\lambda}^{\prime}$ is a solution of

$$
\mathcal{A} u_{0}-\frac{\lambda}{1+h^{\prime}\left(\frac{1}{p}\|u\|_{1, p}^{p}\right)} G u_{0}=0
$$

On the other hand, because $\lambda \in\left(\lambda_{1}, \lambda_{1}+\delta\right)$ and $\lambda_{1}$ is the first eigenvalue of $\left(P_{0}\right)-(E)$, we may have nontrivial critical points of $\Phi_{\lambda}$ only if

$$
h^{\prime}\left(\frac{1}{p}\|u\|_{1, p}^{p}\right)=\frac{\lambda}{\lambda_{1}}-1
$$

Thanks to the definition of $h$, we deduce that $\frac{1}{p}\|u\|_{1, p}^{p} \in(1,3)$. Since $\lambda_{1}$ is simple, we deduce that $u_{0}$ is collinear to $u_{1}$ with constant of proportionality $m$ belonging to the interval $\left(p^{\frac{1}{p}},(3 p)^{\frac{1}{p}}\right)$. Here $u_{1}$ is the positive and normalized eigenfunction associated to $\lambda_{1}$. Then, for $\lambda \in\left(\lambda_{1}, \lambda_{1}+\delta\right), \Phi_{\lambda}^{\prime}$ has precisely three isolated critical points $-m u_{1}, 0, m u_{1}$, for some positive constant $m$.

To achieve the proof we claim the following assertions:

Claim 1: $\Phi_{\lambda}$ is weakly lower semi-continuous.

Claime 2: $\Phi_{\lambda}$ is coercive, that is,

$$
\lim _{\|u\|_{1, p} \rightarrow+\infty} \Phi_{\lambda}(u)=\infty .
$$

Indeed, in order to prove Claim 1, we process as follows. Lemma 3.3 implies

$$
\left\langle G u_{n}, u_{n}\right\rangle \longrightarrow\langle G u, u\rangle .
$$

Now, since the norm is weak lower semicontinuous, we have

$$
\|u\|_{1, p}=\lim _{n \rightarrow+\infty}\left\|u_{n}\right\|_{1, p} .
$$

Therefore, since $h$ is increasing in the interval $(3, \infty),(4.8)$ and (4.9) lead to

$$
\lim _{n \rightarrow \infty} \Phi_{\lambda}\left(u_{n}\right) \geq \Phi_{\lambda}(u) .
$$

To prove the Claim 2, we argue as follows. Due to (4.4), we can evaluate $\Phi_{\lambda}(u)$. In fact,

$$
\begin{aligned}
\Phi_{\lambda}(u) & =\frac{1}{p}\langle\mathcal{A} u, u\rangle-\frac{\lambda_{1}}{p}\langle G u, u\rangle+\frac{\lambda_{1}-\lambda}{p}\langle G u, u\rangle+h\left(\frac{1}{p}\|u\|_{1, p}^{p}\right) \\
& \geq \frac{\lambda_{1}-\lambda}{p}\langle G u, u\rangle+h\left(\frac{1}{p}\|u\|_{1, p}^{p}\right) .
\end{aligned}
$$

Thus, for $\|u\|_{1, p}$ being sufficiently large, we obtain via the definition of the function $h$ that

$$
\begin{aligned}
\Phi_{\lambda}(u) & \geq \frac{\lambda_{1}-\lambda}{p}\langle G u, u\rangle+\frac{1}{p}\|u\|_{1, p}^{p} \\
& \geq \frac{\lambda_{1}-\lambda}{p}\langle G u, u\rangle+a\left(\frac{1}{p}\|u\|_{1, p}^{p}-2\right) .
\end{aligned}
$$

Hence, due to the fact that $\lambda_{1}<\lambda$, we obtain

Consequently,

$$
\Phi_{\lambda}(u) \geq \frac{\lambda_{1}-\lambda}{p \lambda_{1}}\|u\|_{1, p}^{p}+a\left(\frac{1}{p}\|u\|_{1, p}^{p}-2\right) .
$$

By the choice of $a$, we can verify easily

$$
\left.\Phi_{\lambda}(u) \geq \frac{1}{p}\left(\frac{\lambda_{1}-\lambda}{\lambda_{1}}+a\right)\|u\|_{1, p}^{p}-2 a\right) .
$$

$$
\frac{\lambda_{1}-\lambda}{\lambda_{1}}+a>\frac{\lambda_{1}-\lambda}{\lambda_{1}}+\frac{\delta}{\lambda_{1}}>0
$$


Therefore, $\Phi_{\lambda}(u) \rightarrow \infty$, as $\|u\|_{1, p} \rightarrow \infty$. Then the claim 2 is proved.

Now, $\Phi_{\lambda}$ has precisely two points at which the minimum of $\Phi_{\lambda}$, because it is even. Moreover, this minimum is achieved at : $-m u_{1}$ and $m u_{1}$ for vertain $m \in(0, \infty)$. However origin 0 is of "saddle" type isolated critical point. Now, Lemma 3.5 gives

$$
\operatorname{Ind}\left(\Phi_{\lambda}^{\prime},-m u_{1}\right)=\operatorname{Ind}\left(\Phi_{\lambda}^{\prime}, m u_{1}\right)=1
$$

and

$$
\left\langle\Phi_{\lambda}^{\prime} u, u\right\rangle>0
$$

whenever $u \in \partial B_{R}(0)$, with $R$ is large enough. In fact, we may check that

$$
\langle\mathcal{A} u, u\rangle>3 \text { and }\langle\mathcal{A} u, u\rangle>\lambda\langle G u, u\rangle \text {. }
$$

Then

$$
\begin{aligned}
\left\langle\Phi_{\lambda}^{\prime}(u), u\right\rangle & \geq\left(\lambda_{1}-\lambda\right)\langle G u, u\rangle+a\langle\mathcal{A} u, u\rangle \\
& \geq \frac{-\delta}{\lambda_{1}}\langle\mathcal{A} u, u\rangle+a\langle\mathcal{A} u, u\rangle \\
& \geq\left(a-\frac{\delta}{\lambda_{1}}\right)\|u\|_{1, p}^{p} .
\end{aligned}
$$

This implies that $\left\langle\Phi_{\lambda}^{\prime}(u), u\right\rangle \rightarrow \infty$, as $\|u\|_{1, p} \rightarrow \infty$ by definition of $h$.

We deduce from Lemma 3.6 that

$$
\operatorname{Deg}\left[\Phi_{\lambda}^{\prime} ; B_{R}(0), 0\right]=1
$$

We consider the radius $R$ so that $\alpha u_{1} \in \partial B_{R}(0)$.

Since the degree is additive and taking in account (4.10) and (4.11), we conclude that

$$
\operatorname{Deg}\left[L-\lambda G ; B_{\epsilon}(0), 0\right]=-1 \text {. }
$$

Also we have

whenever $\|u\|_{1, p} \rightarrow 0$. Therefore we get

$$
\langle\mathcal{A} u, u\rangle-\lambda\langle G u, u\rangle \rightarrow 0
$$

$$
\operatorname{Deg}\left(\mathcal{A} u-\lambda G, B_{\epsilon}(0), 0\right)=\operatorname{Ind}\left(\Phi_{\lambda}^{\prime}, 0\right),
$$

for $\epsilon>0$ sufficiently small enough. Finally (2.18) and the invariance principle of the degree permit us to deduce that

$$
\operatorname{Deg}\left(T_{\lambda}, B_{\epsilon}(0), 0\right)=\operatorname{Deg}\left(\mathcal{A} u-\lambda G, B_{\epsilon}(0), 0\right),
$$

for any $\lambda$ near to $\lambda_{1}$ but $\lambda \neq \lambda_{1}$. Finally, (4.6) and (4.10) - (4.13) imply that

$$
\begin{gathered}
\operatorname{Deg}\left(T_{\lambda}, B_{\epsilon}(0), 0\right)=1 \text { for } \lambda \in\left(\lambda_{1}-\delta, \lambda_{1}\right) \text { and } \\
\operatorname{Deg}\left(T_{\lambda}, B_{\epsilon}(0), 0\right)=-1 \text { for } \lambda \in\left(\lambda_{1}, \lambda_{1}+\delta\right) .
\end{gathered}
$$

for $\epsilon>0$ sufficiently small. The "jump" of the degree is stated and the proof of the theorem is achieved.

\section{References}

[1] Adams, R. A.: Sobolev spaces, Academic Press, New York, 1975.

[2] Anane, A.: Simplicité et isolation de la première valeur propre du p-laplacien avec poids, Comptes Rendus de lAcadémie des Sciences-Series I-Mathematics 305 16(1987), 725728.

[3] Atkinson, C., Champion, C. R.: Some boundary problems for the equation $\nabla \cdot\left(|\nabla \phi|^{N} \nabla \phi\right)=0$, Q. J. Mech. Appl. Math. 37(1983), 401-419.

[4] Atkinson, C., Jones, C. W.: Similarity solutions in some nonlinear diffusion problems and in boundarylayer flow of a pseudo plastic fluid, Q. J. Mech. Appl. Math. 27(1974), 193-211.

[5] Babuska, I., Osborn, J.: Eigenvalue problems, in "Handbook of numerical analysis", Vol. II, NorthHolland, Amsterdam, (1991), 314-787.

[6] Beurling, A., Livingston, A.: A theorem on duality mappings in Banach space, Ark. Mat. 4(1962), 405-411. 
[7] Binding, P. A., Huang, Y. X.: Bifurcation from eigencurves of the p-Laplacian, Diff. Int. Equations 8 no. 2 (1995), 415-418.

[8] Browder, F. E.: On a theorem of Beurling and Livingston, Cand. J. Math. 17(1965), 367-372.

[9] Browder, F. E., Fixed point theory and nonlinear problems, Bull. Amer. Math. Soc. 9(1983), 1-39.

[10] Browder, F. E., Petryshyn, W. F.: Approximation methods and the generalized topological degree for nonlinear mapping in Banach spaces, J. Funct. Anal. 3(1969), 217-245.

[11] Bronder, J. F., Rossi, J. D.: A nonlinear eigenvalue problem with indefinite weights related to Sobolev trace embedding, Publ. Mat. 46(2002), 221-235.

[12] Crîngnu, J.: Variational and topological methods for Neumann problems with p-Laplacian, Communications on Applied Nonlinear Analysis 11(2004), 1-38.

[13] Ciorănascu, I.: Duality mapping in nonlinear functional analysis, Publishing House of Romanian Academy Bucharest, 1974. (in Romanian).

[14] Del Pino, M. A., Manesevich, R. F.: Global bifurcation from the eigenvalues of the p-Laplacian, J. Diff. Equations 130(1996), 235-246.

[15] De Thélin,F.: Sur l'espace propre associé à la première valeur propre du pseudo-laplacien, Comptes Rendus Mathmatique. Académie des Sciences. Paris, Sér. I Math., 303(1986), 355-358.

[16] Diaz, J. I., Nonlinear partial differential equations and free boundaries, Vol.I, Elliptic Equations, London, 1985.

[17] Drábek, P., El khalil, A., Touzani A., A result on the bifurcation from the principal eigenvalue of the $A_{p}$-Laplacian, Abstract and Applied Analysis, vol. 2, Nos. 3-4 (1997), 185-195.

[18] Drábek, P., El khalil, A., Touzani A., A bifurcation Problem for the Principal Eigencurve of the pLaplacian, Applicable Analysis, vol. 72, Nos. 3-4 (1999), 399-410.

[19] Drábek, P., Huang, Y. X., Bifurcation problems for the p-Laplacian in $\mathbb{R}^{n}$, Trans-Amer, Math. Soc. 349(1997), 171-188.

[20] Edmunds, D.E., Evans, W.D., Spectral Theory and Differential Operators, Clarendon Press, University Oxford Press, New York, 1987.

[21] El khalil, A., Touzani A.: On the first eigencurve of the p-Laplacian, Partial Differential Equations, Lecture Notes in Pure and Applied Mathematics Series, Marcel Dekker, Inc. 229(2002), 195-205.

[22] Gilbarg, D., Trudinger, N.: Elliptic Partial Differential Equations of Second order, Springer, Berlin, 1983.

[23] Lindqvist, P.: On the equation $\operatorname{div}\left(|\nabla u|^{p-2} \nabla u\right)+\lambda|u|^{p-2} u=0$, Proc. Amer. Math. Soc. 109(1990), 157-164.

[24] Pao, C. V.: Nonlinear parabolic and elliptic equations, Plenum Press, New York, London, 1992.

[25] Pelissier, M. C.: Sur quelques problèmes non linéaires en glaciologie, Thèse, Publications Mathématiques d'Orsay, No. 110, 1975.

[26] Philip, J. R.: N-diffusion, Aust. J. Phys. 14(1961), 1-13.

[27] Rabinowitz, P. H.: Some global results for nonlinear eigenvalue problem, J. Funct. Anal. 7(1971), 487513.

[28] Skrypnik, I. V.: Nonlinear Elliptic Boundary Value Problem, Teubner, Leipzig, 1986. 